\title{
Structural feature factors extraction of upper body shape for young female
}

\author{
Yujie Ren, Hongshu Jin* \\ Wuhan textile university, Wuhan, Hubei 430073, China
}

\begin{abstract}
Human body shape feature points are the key information and basic unit for human body model we are constructing, which performance the difference of body shapes. The purpose of the study is to extract the structural feature factors related to the upper body surface feature points for young females. The 12 feature points of upper body surface were manually confirmed from anthropometric expertise. A total of 31 measurements items, including 3 body surface measurement and 28 photo measurement, were collected for 33 females college students According to the results of correlation analysis, the feature variables of the width, thickness and height dimension based on 12 feature points significantly respectively correlated to the variables of their coordinate orientation, furthermore, the correlated relationship which reflected the width and height features of neck and shoulders shape mainly affected by local skeletal structures. Then, four principle component factors account for upper body shapes of young females, such as width, thickness, height and shoulder shape with characteristic value all over 1 , were extracted by the principal component analysis, and the cumulative contribution rate reached $87.387 \%$. Therefore, a total of 8 feature variables sifted from each principle component factor with a loading coefficient over 0.7 as fundamental typical indicators represent the three-dimensional characteristics of body surface feature points reflecting the divergence of body shapes, and it is useful structural information for individual human body modelling.
\end{abstract}

\section{Introduction}

Under the background of the intelligent development of the clothing industry and the trend of personalized clothing consumption, virtual human modelling has become a hotspot in the field of multidisciplinary cross-application research. The human body surface feature points (measurement points) are the key information reflecting the differences of human body shapes, and also the basic parametric information for subdividing and identifying the human body shape.

In the field of theoretical research, the body shape classification based on diverse analysis of human body parameters, such as the square ratio of cross-section's area and girth on $\mathrm{BL}, \mathrm{WL}$ and $\mathrm{HL}^{[1]}$, and the descriptions of the differences of trunk longitudinal section $^{[2]}$, supplied and refined the national standard body type classifications. Furthermore, the studies on the body local morphology subdivision ${ }^{[3-4]}$ proposed diversified human body morphological parameters based on feature points, as well as the method of morphological information combination for representing the difference of body shape.

On the method of human body shape measurement, non-contact $3 \mathrm{D}$ human body scanning and $2 \mathrm{D}$ photo measurement were commonly used for collecting the data by the human body surface features points labelled manually currently. However, the original technical problems of 3D human body scanning, such as the high cost of labor, the local data missing, and the deformation of automatic local shape renovation, limit its application in individual human body modelling accurately, as well as in the field of clothing mass customization $^{[5]}$. In addition, the two-dimensional human body image is used as a reliable data source for obtaining human body shape feature values ${ }^{[6]}$, describing and identifying important information of the human body shape differences.

In this study, it is aiming to extract the structural feature factors related to the upper body surface feature points for young female. For unified body measurement standard, the identification of body surface feature points was confirmed from manual measuring methods. And the extracted structural feature factors were the important references for typical indices selecting represent the three-dimensional characteristics of body surface feature points reflecting the divergence of body shapes, and it is useful structural information for individual human body modelling.

\section{Method of measurement data acquisition}

\footnotetext{
* Corresponding author: 2013025@wtu.edu.cn
} 


\subsection{Measurement object}

The measurement subjects were 33 female college students aged 18-23.

\subsection{Definition of body surface feature points}

The 12 upper body surface feature points were confirmed from anthropometric expertise manually and showed in Figure 1.

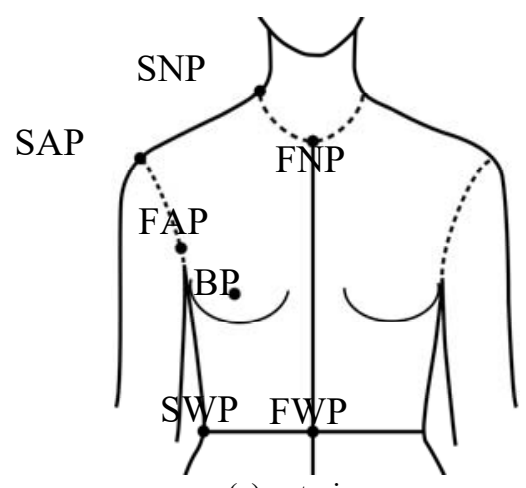

(a) anterior

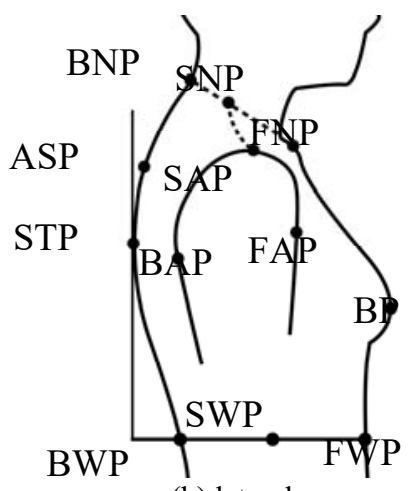

(b) lateral

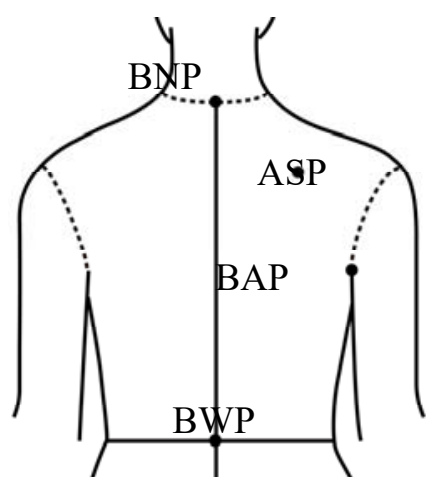

(c) posterior

Figure 1. Feature points on body surface.

Among them, the feature points such as SNP (side neck point), FNP (front neck point), BNP (back neck point), SAP (shoulder acromion point), BP (bust point) adopt the general definition of national standard GB/T 16160-2008 "Anthropometric definition and methods for garment". For the definitions, the other feature points are referred to the ergonomic measurement point definition by Machiko MIYOSHI ${ }^{[7]}$, including the armpit front/back point is the extreme point of skin fold in front of and behind the trunk and upper arm which is simple and easy to confirm.

\subsection{Photography and measurement methods}

The subjects were required to wear fitted underwear, keep upright and look ahead.

The 12 feature points and related reference lines were marked using triangular markers on the body surface.

The anterior, lateral and posterior photos for each subject were shot using Canon EOS 5D Mark II SLR camera. The shooting distance was $10 \mathrm{~m}$, and the height of centre of lens was $1 \mathrm{~m}$, which was roughly the same as the height of waist. At the same time, a pole marked $50 \mathrm{~cm}$ was placed on the right side of subject horizontally as a reference for converting and recording the actual measurement. The photo for measuring is shown in Figure 2.

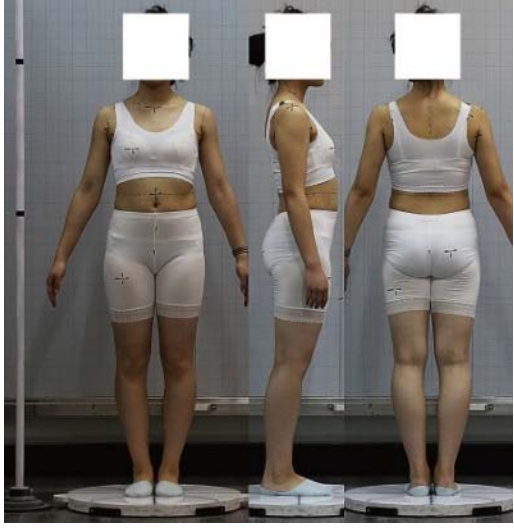

Figure 2. Photo used for measuring.

\subsection{Upper body measurement items}

There is a total of 31 items for the upper body measurement, including 28 photo measurement items and 3 body surface measurements (upper body control area, bust, waist, height).

The photo measurement items show as Figure 3, including 7 items of front/back width measurement (Figure 3(a)), 12 items of thickness measurement (Figure 3(b)), and 9 items of height measurement (Figure 3(c)).

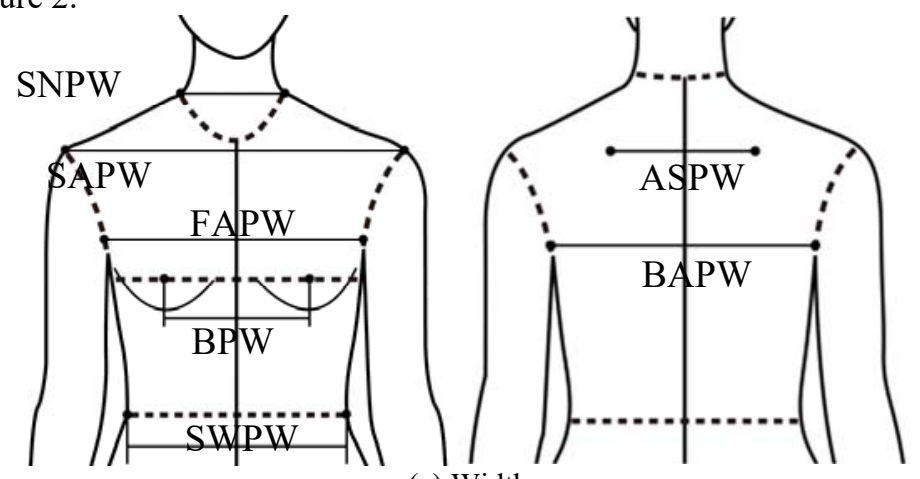

(a) Width 


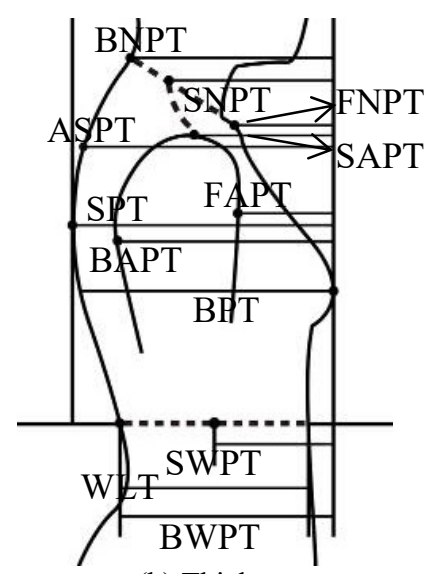

(b) Thickness

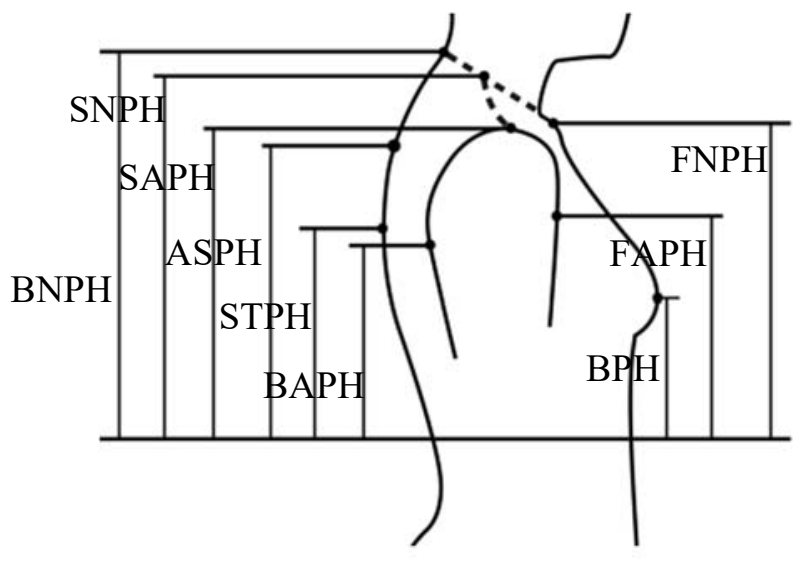

(c)Height

Figure 3. Schematic diagram of measurement items.

\section{Extraction of upper body features of young women}

For extracting the morphological feature factors of the upper body of young women, the statistical analysis method of Kolmogorov-Smirnov test, correlation analysis and principle component analysis were utilized by IBM SPSS Statistics 21 data analysis software.

\subsection{Upper body data distribution test}

According to the results of Kolmogorov-Smirnov test, the upper body morphologic feature variables all follow the normal distribution.

Taking the size of A's upper body control part (height, bust and waist) in GB/T+1335.2-2008 "Standard sizing for garment-Woman" as the test value, the One-Samples T-Test were done and the results shown in Table 1.

Table 1. Mean test of sample female youth and national standard female data.

\begin{tabular}{lccccc}
\cline { 2 - 6 } & Project & Mean $(\mathrm{cm})$ & Standard deviation $(\mathrm{cm})$ & Test value $(\mathrm{cm})$ & Sig.(2-tailed) \\
\cline { 2 - 6 } & height & 162.67 & 4.75 & 160 & $* * \mathrm{a}$ \\
bust & 82.17 & 5.21 & 84 & $* * \mathrm{a}$ \\
Waist & 65.25 & 5.80 & 68 & N.S. ${ }^{\mathrm{b}}$ \\
\cline { 2 - 6 } a $* * \mathrm{P}<0.01$. & & & &
\end{tabular}

The average height and bust of the subjects taken in this study showed significant differences with standard size. Compared with the national average, the average height and bust girth were slightly higher lower respectively.

\subsection{Relationship between upper body feature variables}

\subsubsection{Correlation relationship between surface morphological characteristics variables.}

The results of correlation analysis of upper body surface morphological feature variables showed that: 1) For the width variables, SWPW(WL width) showed a highly significant correlation with FAPW(armpit front fold point width) and BAPW(armpit back fold point width), respectively; SAPW (shoulder acromion point width ) and SNPW(side neck point )showed a correlation between $\mathrm{r}=0.6$ and above, reflecting the characteristics of the shoulder and neck width; The correlation coefficient between ASPW(suprascapular point width) and other width characteristic variables was lower than $r=0.6$, showing the width characteristic based on the bone structure of the shoulder.

2) Among height variables, the correlation coefficient between SNPH(side neck point height), BNPH(back neck point height) and FNPH(front neck point height) reached more than $r=0.85$.SAPH(shoulder acromion point height); BAPH(armpit back fold point height) and ASPH (suprascapular point height) showed a high correlation above $\mathrm{r}=0.8$, showing the height characteristics of neck and shoulder respectively.

3) Among the thickness variables, the correlation coefficient between SNPT(side neck point thickness), FNPT(front neck thickness) and BNPT(back neck point thickness) were above $\mathrm{r}=0.9$; The correlation coefficients between SAPT(shoulder acromion point thickness), FAPT(armpit front fold point thickness), BAPT (armpit back fold point thickness) and ASPT (suprascapular point width) were more than $r=0.8$; The correlation coefficient between waist thickness and BPT(chest thickness) and STPT(body thickness) is close to $r=0.9$, which describes the thickness characteristics of neck-shoulder and bust-waist respectively. 
To sum up, the width, height and thickness variables of upper body surface morphology showed high significant correlations respectively, and the main morphological features of shoulder, neck and trunk cadres were also shown.

\subsubsection{Relationship between basic size and characteristic variables.}

Bust and waist were significantly correlated with SWPW(WL width), BPT(bust thickness), WLT(WL thickness) and other variables over $\mathrm{r}=0.8$, indicating the close relationship between the shape characteristics of upper body bust and waist and the size of circumference.

In addition, FAPW(armpit front fold point width), BAPW(armpit back fold point width) and SWPW(point width) were significantly correlated with BPT(bust thickness), STPT(body thickness) and WLT(WL thickness) respectively, and the relationship between upper body morphology and local characteristics was described.

The correlation coefficient between BPW (bust point width) and other morphological characteristic variables is lower than $\mathrm{r}=0.5$, and the position of bust point is greatly affected by the shape and material of bra cup, showing no correlation with morphological characteristics.

\subsection{Extraction of principal components of surface morphological features}

Based on the results of correlation analysis, excluded variables unrelated and repetitive, 19 morphological characteristic variables are screened out and used for the principle component analysis. The results of principal component analysis are shown in Table 2. four principal components with eigenvalues above 1 were extracted by the maximum variance factor rotation method, and the cumulative variance reached $87.387 \%$.

Table 2. Extraction of morphological features of upper body after rotation.

\begin{tabular}{|c|c|c|c|c|}
\hline \multirow{2}{*}{ Characteristics of the variables } & \multicolumn{4}{|c|}{ Ingredients } \\
\hline & 1 & 2 & 3 & 4 \\
\hline FAPT & .958 & & & \\
\hline SAPT & .912 & & & \\
\hline ASPT & .863 & & & \\
\hline BAPT & .846 & & & \\
\hline BNPT & .839 & & & \\
\hline FNPT & .831 & & & \\
\hline STPT & .791 & & & \\
\hline BPT & .683 & & & \\
\hline BAPH & & .950 & & \\
\hline ASPH & & .933 & & \\
\hline SAPH & & .933 & & \\
\hline $\mathrm{BNPH}$ & & .887 & & \\
\hline FNPH & & .871 & & \\
\hline BAPW & & & .842 & \\
\hline WLT & & & .821 & \\
\hline BWPT & & & .807 & \\
\hline SWPW & . & & .782 & \\
\hline SAPW & & & & .891 \\
\hline ASPW & & & & .761 \\
\hline Eigenvalue & 6.283 & 4.346 & 4.217 & 1.758 \\
\hline Explain the variance (\%) & 33.069 & 22.873 & 22.195 & 9.251 \\
\hline Cumulative variance $(\%)$ & 33.069 & 55.942 & 78.136 & 87.387 \\
\hline
\end{tabular}

1) According to the 8 variables related to the thickness of upper body show over 0.6 high loading coefficient, the first principal component could be interpreted as trunk and shoulder-neck thickness factor. Inside, BWPT (WL back centre point thickness) and BNPT (back neck point thickness) and STPT (body thickness) in the first principal component expressed lateral dorsal curve morphology. In particular, BNPT (back neck point thickness) and SAPT(shoulder acromion point thickness), expressed the lateral morphological characteristics of neck and shoulder characteristic, and STPT (body thickness) expressed the lateral morphological characteristics of trunk, which were the typical indictors of thickness factor.
2) In consideration of height variables with over 0.7 high loading coefficient in the second principal component, it could be interpreted as upper body height factor. Inside, BNPH (back neck point) and SAPH(shoulder acromion point height) explained the height characteristics of neck and shoulder. Meanwhile, BNPH (back neck point height) also expresses the height characteristics of trunk. These variables were selected as typical indictors of the second principle component.

3) The third and fourth principal component includes 4 and 2 variables with above 0.7 high loading coefficient variables which described the trunk shape characterises and shoulder-width characterises, it could be interpreted as waist shape factor and shoulder-width 
factor, respectively. SAPW (shoulder acromion point width), SWPW (WL width) and BWPT(WL back centre point thickness) of the third and fourth principal components are respectively typical features to express shoulder width and waist shape.

The above principal components include the thickness, height and width of the trunk, shoulders and neck, and comprehensively express the relationship between the characteristic variables of three azimuths on the body surface. In particular, the anthropometry definition of BNP(back neck point) and SAP(shoulder acromion point) is clear, and the influence of manual operation error is small. The related factors reflect the main characteristics of the shape of human shoulder and neck.

And then, a total of 8 typical indexes describing the overall and local morphological characteristics of human body were extracted from the three azimuths of thickness, height and width. Collinearity detection results of typical characteristic variables in each principal component showed that VIF (Variance Inflation Factor) among typical indicators was less than 10. Therefore, statistically, the extracted 8 typical indicators were reasonable.

\section{Conclusion and future work}

The aim of this study is to extract the structural feature factors related to the upper body surface feature points for young females. Based on 12 surface feature points of the human body, human body feature measurements were collected. The main results of this study summarized as follows:

1) the relationship between each feature size was obtained through correlation analysis.

2) principal component analysis was used to extract 8 typical feature indexes that can describe the basic feature information of the human body.

In future work, the author plans to increase the sample size and continue to use the approach for morphological subdivision and body modelling to promote the development of clothing customization.

\section{References}

1. Wang Q M 2016 Female body classification in Jiangsu and Zhejiang based on cross-sectional area of body J. Journal of Textile Research. vol 37, no 05. 131-136

2. Xia F Q, Wu G, Xie H Y and Zhong Y 2017 Classification of body shape based on longitudinal section curve J. Journal of Textile Research. vol 38, no 06. 86-91

3. Hamad M, Thomassey S and Bruniaux P 2017 A new sizing system based on $3 \mathrm{D}$ shape descriptor for morphology clustering J. Computers \& Industrial Engineering. vol 113. 683-692

4. Sun N L and Song X X 2014 Research on chest classification of young women in Shanghai J. Journal of Silk. vol 51, no 09. 34-39
5. Ni S M, Jin J F and Pang C F 2014 Research of young female somatotype based on longitudinal profile J. Textile Research. vol 35, no 08. 87-93

6. Luo S H and Wang J P 2013 Research on 2-D image-based non-contact anthropometric technology J. Journal of Textile Research. vol 34, no $08.151-155$

7. Milyoshi M 2000 The Theory of Fashion Modeling, 1st ed (Beijin: China Textile \& Apparel Press) p67 